\title{
RICHNESS OF AVIFAUNA IN GOREWADA INTERNATIONAL BIO-PARK, NAGPUR, CENTRAL INDIA
}

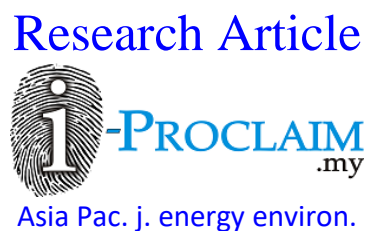

\section{Virendra A. Shende ${ }^{1}$, Kishor G. Patil ${ }^{2}$}

\author{
${ }^{1}$ Assistant Professor, Department of Zoology, K. Z. S. Science College, Bramhani-Kalmeshwar, Dist- Nagpur (M.S.), INDIA \\ ${ }^{2}$ Associate Professor, Department of Zoology, Govt. Institute of Science, R. T. Marg, Nagpur (M.S.), INDIA \\ *Email for Correspondence: virushende@gmail.com
}

Abstract

The present study comprises the biodiversity of birds in Gorewada International bio-park situated at north-west of Nagpur city with geographical location $21^{\circ} 11^{\prime} \mathrm{N} 79^{\circ} 2^{\prime} \mathrm{E}$ and a good habitat for avian biodiversity. Bird watching and recording were done from March 2011 to February 2014, in the morning and evening hours during Sunday and holidays. 190 species belonging to 128 genera, 51 families and 17 orders (Podicipediformes, Pelecaniformes, Ciconiiformes, Anseriformes, Falconiformes, Galliformes, Gruiformes, Charadriiformes, Columbiformes, Psittaciformes, Cuculiformes, Strigiformes, Caprimulgiformes, Apodiformes, Coraciiformes, Piciformes and Passeriformes) were recorded. The order Passeriformes is the largest order belonging to 20 families and 75 bird species. Out of total bird species, $89(46.84 \%)$ are resident, $77(40.53 \%)$ are resident migrant and $24(12.63 \%)$ are migratory species.

Forest region of this bio-park provides heterogeneity in vegetation and lake is rich in aquatic fauna and other food for avian species. Therefore, this region is well suitable for feeding, resting and roosting site for aves. The report obtains information regarding resident birds, migratory birds and resident migratory birds of Gorewada bio-park.

Key words

Avifauna, Birds, Gorewada, International bio-park, Biodiversity

This article is is licensed under a Creative Commons Attribution-NonCommercial 4.0 International License.

Attribution-NonCommercial (CC BY-NC) license lets others remix, tweak, and build upon work non-commercially, and although the new works must also

acknowledge \& be non-commercial.

\section{INTRODUCTION}

The Indian subcontinent is very rich in biodiversity; contains about 1,300 species or over $13 \%$ of the world's birds (Grimmett et al, 1998). The recent studies of freshwater biodiversity and wetlands are found to be the richest biodiversity sites by holding major share of the existing avifauna. Freshwater wetland support $20 \%$ of the known range of biodiversity in India. Avifauna is an important for the ecosystem as they play various roles as scavenger, pollinators and predators of insect pest (Padmavati et al. 2010). The changes in environment like urbanization and industrialization disturbs the avian habitats (Sanjay, 1993).

Gorewada is the developing international bio-park in north-west of Nagpur city and its geographical location is $21^{\circ} 11^{\prime} \mathrm{N} 79^{\circ} 2^{\prime} \mathrm{E}$. It provides good habitat for avian species and some wild life. The park is basically divided into African Safari, Biopark, Energy Plaza, Trails, Indian Safari, Hight Safari, Rescue Safari and Gorewada Reservoir. Gorewada reservoir is bordered by thick forest on three sides. It was developed by the water works department, in 1912 as the primary drinking water source for Nagpur. The lake was once known as Sita Gondi, later it named after Gorewada village. Its catchment area is approx. 11 sq. mile $(17,702.74 \mathrm{sqmt})$. In spite of its global significance; studies of avifauna of Gorewada International Park have not been clearly undertaken.

The present work has been carried out the checklist, occurrence and richness of bird species inhibiting the Gorewada International Park. 


\section{Material AND Methods}

The present study has been carried out for a period of two year from March 2011 to February 2013. Bird watching and recording were done during Sunday and holidays in such a way that there should be least one visit in the study area during a month. The observations were made with the aid of a digital cameras and binoculars.

The Point Transects Method (Gregory et al. 2004) was adapted to study the bird population. The sites were visited in morning and evening hours to note maximum possible species of birds and to record its activities. Sometimes afternoon visits were made to observe the bird activities; particularly in winter season when the migratory species reported in the study area. The recorded species are identified with the help of photographs and by using standard books such as Ali (2001), Animal Life Encyclopedia and various publications. The checklist and catalogs of the avian species are prepared.

\section{Observations}

In the present study, we recorded 190 species of birds belonging to 128 genera, 51 families and 17 orders. The order Podicipediformes, Pelecaniformes, Ciconiiformes, Anseriformes, Falconiformes, Galliformes, Gruiformes, Charadriiformes, Columbiformes, Psittaciformes, Cuculiformes, Strigiformes, Caprimulgiformes, Apodiformes, Coraciiformes, Piciformes and Passeriformes were consisted of 01 family, 02 genera and 02 species; 01 family, 02 genera and 04 species; 03 families, 15 genera and 19 species; 01 family, 07 genera and 16 species; 01 family, 08 genera and 10 species; 01 family, 03 genera and 04 species; 01 family, 04 genera and 05 species; 07 families, 12 genera and 19 species; 01 family, 03 genera and 07 species; 01 family, 01 genus and 03 species; 01 family, 07 genera and 09 species; 02 families, 02 genera and 02 species; 01 family, 01 genus and 01 species; 02 families, 02 genera and 02 species; 05 family, 07 genera and 08 species; 02 family, 04 genera and 04 species and 20 families, 48 genera and 75 species respectively (Table 1 and Table 3).

Table 1: Checklist of Avifauna of Gorewada International Bio-Park

\begin{tabular}{|c|c|c|c|c|c|}
\hline S. N. & Order & Family & Scientific Name & Common Name & Status \\
\hline 1. & $\begin{array}{l}\text { Podicipediformes } \\
\text { (1 Family) }\end{array}$ & $\begin{array}{l}\text { Podicipedidae } \\
(2 \text { Genera })\end{array}$ & $\begin{array}{l}\text { Podicepscristatus } \\
\text { Tachybaptusruficollis }\end{array}$ & $\begin{array}{l}\text { Great Crested Grebe } \\
\text { Little Grebe }\end{array}$ & $\begin{array}{l}\mathrm{M} \\
\mathrm{R}\end{array}$ \\
\hline 2. & $\begin{array}{l}\text { Pelecaniformes } \\
\text { (1 Family) }\end{array}$ & $\begin{array}{l}\text { Phalacrocoracidae } \\
\text { (2 Genera) }\end{array}$ & $\begin{array}{l}\text { Anhinga melanogaster } \\
\text { Phalacrocoraxcarbo } \\
\text { Phalacrocoraxfuscicollis } \\
\text { Phalacrocoraxniger }\end{array}$ & $\begin{array}{l}\text { Darter } \\
\text { Great Cormorant } \\
\text { Indian Cormorant } \\
\text { Little Cormorant }\end{array}$ & $\begin{array}{l}\text { RM } \\
\text { RM } \\
\text { R } \\
\text { RM }\end{array}$ \\
\hline \multirow[t]{3}{*}{3.} & \multirow[t]{3}{*}{$\begin{array}{l}\text { Ciconiiformes } \\
\text { (3 Families) }\end{array}$} & $\begin{array}{l}\text { Ardeidae } \\
\text { (13 Genera) }\end{array}$ & $\begin{array}{l}\text { Anastomusoscitans } \\
\text { Ardeacinerea } \\
\text { Ardeapurpurea } \\
\text { Ardeolagrayii } \\
\text { Bubulcus ibis } \\
\text { Butoridesstriata } \\
\text { Casmerodiusalbus } \\
\text { Ciconianigra } \\
\text { Dupetorflavicollis } \\
\text { Egrettagarzetta } \\
\text { Egrettaintermedia } \\
\text { Ixobrychuscinnamomeus } \\
\text { Ixobrychus minus } \\
\text { Ixobrychussinensis } \\
\text { Mesophoyxintermedia } \\
\text { Mycterialeucocephala } \\
\text { Nycticoraxnycticorax }\end{array}$ & $\begin{array}{l}\text { Asian Open bill } \\
\text { Grey Heron } \\
\text { Purple Heron } \\
\text { Indian Pond Heron } \\
\text { Cattle Egret } \\
\text { Little Heron } \\
\text { Large Egret } \\
\text { Black Stork } \\
\text { Black Bittern } \\
\text { Little Egret } \\
\text { Smaller Egret } \\
\text { Chesnut Bittern } \\
\text { Little Bittern } \\
\text { Yellow Bittern } \\
\text { Median Egret } \\
\text { Painted Stork } \\
\text { Black-crowned Night Heron }\end{array}$ & $\begin{array}{l}\text { RM } \\
\text { RM } \\
\text { RM } \\
\text { R } \\
\text { R } \\
\text { R } \\
\text { R } \\
\text { M } \\
\text { RM } \\
\text { R } \\
\text { R } \\
\text { RM } \\
\text { M } \\
\text { RM } \\
\text { R } \\
\text { RM } \\
\text { RM }\end{array}$ \\
\hline & & Ciconiidae (1 Genus) & Ciconiaepiscopus & Woolly-necked Stork & RM \\
\hline & & $\begin{array}{l}\text { Threseskiornithidae } \\
\text { (1 Genus) }\end{array}$ & Pseudibispapillosa & Black Ibis & RM \\
\hline 4. & $\begin{array}{l}\text { Anseriformes } \\
\text { (1 Family) }\end{array}$ & $\begin{array}{l}\text { Anatidae } \\
(7 \text { Genera })\end{array}$ & $\begin{array}{l}\text { Anasacuta } \\
\text { Anasclypeata } \\
\text { Anascrecca } \\
\text { Anaspenelope } \\
\text { Anasplatyrhynchos } \\
\text { Anaspoecilorhyncha } \\
\text { Anasquerquedula }\end{array}$ & \begin{tabular}{|l} 
Northern Pintail \\
Northern Shoveler \\
Common Teal \\
Eurasian Wigeon \\
Mallard \\
Spot-billed Duck \\
Garganey
\end{tabular} & $\begin{array}{l}\mathrm{M} \\
\mathrm{M} \\
\mathrm{M} \\
\mathrm{M} \\
\mathrm{M} \\
\mathrm{M} \\
\mathrm{M}\end{array}$ \\
\hline
\end{tabular}




\begin{tabular}{|c|c|c|c|c|c|}
\hline S. N. & Order & Family & Scientific Name & Common Name & Status \\
\hline & & & $\begin{array}{l}\text { Anasstrepera } \\
\text { Aythyaferina } \\
\text { Aythyafuligula } \\
\text { Aythyanyrocha } \\
\text { Dendrocygnajavanica } \\
\text { Falcated Duck } \\
\text { Nettapuscoromandelianus } \\
\text { Rhodonessarufina } \\
\text { Tadornaferruginea }\end{array}$ & $\begin{array}{l}\text { Gadwall } \\
\text { Common Pochard } \\
\text { Tufted Duck } \\
\text { Ferruginous Pochard } \\
\text { Lesser Whistling-duck } \\
\text { Anasfalcata } \\
\text { Cotton Teal } \\
\text { Red-crested Pochard } \\
\text { Ruddy Shelduck }\end{array}$ & $\begin{array}{l}\mathrm{M} \\
\mathrm{M} \\
\mathrm{M} \\
\mathrm{M} \\
\mathrm{RM} \\
\mathrm{M} \\
\mathrm{R} \\
\mathrm{M} \\
\mathrm{M}\end{array}$ \\
\hline 5. & $\begin{array}{l}\text { Falconiformes } \\
\text { (1 Family) }\end{array}$ & $\begin{array}{l}\text { Accipitridae } \\
(8 \text { Genera })\end{array}$ & $\begin{array}{l}\text { Accipiter badius } \\
\text { Accipiter nisus } \\
\text { Aquila rapax } \\
\text { Butasturteesa } \\
\text { Circus aeruginosus } \\
\text { Circus macrorous } \\
\text { Elanuscaeruleus } \\
\text { Milvusmigrans } \\
\text { Pernisptilorhyncus } \\
\text { Spilornischeela } \\
\end{array}$ & $\begin{array}{l}\text { Shikra } \\
\text { Eurasian Sparrowhawk } \\
\text { Tawny Eagle } \\
\text { White-eyed Buzzard } \\
\text { Western Marsh Harrier } \\
\text { Pallid Harrier } \\
\text { Black-shouldered Kite } \\
\text { Black kite } \\
\text { Oriental Honey-buzzard } \\
\text { Crested Serpent Eagle } \\
\end{array}$ & $\begin{array}{l}\text { RM } \\
\text { RM } \\
\text { RM } \\
\text { RM } \\
\text { RM } \\
\text { RM } \\
\text { RM } \\
\text { RM } \\
\text { RM } \\
\text { RM } \\
\end{array}$ \\
\hline 6. & $\begin{array}{l}\text { Galliformes } \\
\text { (1 Family) }\end{array}$ & $\begin{array}{l}\text { Phasianidae } \\
(3 \text { Genera })\end{array}$ & \begin{tabular}{|l|} 
Coturnixcoturnix \\
Francolinuspictus \\
Francolinuspondicerianus \\
Pavocristatus \\
\end{tabular} & $\begin{array}{l}\text { Common Quail } \\
\text { Painted Francolin } \\
\text { Grey Francolin } \\
\text { Indian Peafowl } \\
\end{array}$ & $\begin{array}{l}\mathrm{R} \\
\mathrm{RM} \\
\mathrm{RM} \\
\mathrm{RM} \\
\end{array}$ \\
\hline 7. & $\begin{array}{l}\text { Gruiformes } \\
\text { (1 Family) }\end{array}$ & $\begin{array}{l}\text { Rallidae } \\
\text { (4 Genera) }\end{array}$ & $\begin{array}{l}\text { Amaurornisakool } \\
\text { Amaurornisphoenicurus } \\
\text { Fulicaatra } \\
\text { Gallinulachloropus } \\
\text { Porphyrioporphyrio }\end{array}$ & $\begin{array}{l}\text { Brown Crake } \\
\text { White-breasted Waterhen } \\
\text { Common Coot } \\
\text { Common Moorhen } \\
\text { Purple Moorhen } \\
\end{array}$ & $\begin{array}{l}\mathrm{R} \\
\mathrm{R} \\
\mathrm{R} \\
\mathrm{R} \\
\mathrm{RM}\end{array}$ \\
\hline \multirow[t]{7}{*}{8.} & \multirow[t]{7}{*}{$\begin{array}{l}\text { Charadriiformes } \\
\text { (7 Families) }\end{array}$} & $\begin{array}{l}\text { Jacanidae } \\
(2 \text { Genera })\end{array}$ & $\begin{array}{l}\text { Hydrophasianuschirurgus } \\
\text { Metopidiusindicus }\end{array}$ & $\begin{array}{l}\text { Pheasant tailed Jacana } \\
\text { Bronze winged Jacana }\end{array}$ & $\begin{array}{l}\mathrm{R} \\
\mathrm{R}\end{array}$ \\
\hline & & $\begin{array}{l}\text { Charadriidae } \\
(2 \text { Genera })\end{array}$ & $\begin{array}{l}\text { Charadriusalexandrinus } \\
\text { Charadriusdubius } \\
\text { Charadriusmongolus } \\
\text { Vanellusindicus } \\
\text { Vanellusmalarbaricus } \\
\end{array}$ & $\begin{array}{l}\text { Kentish Plover } \\
\text { Little Ringed Plover } \\
\text { Lesser Sand Plover } \\
\text { Red-wattled Lapwing } \\
\text { Yellow-wattled Lapwing }\end{array}$ & \begin{tabular}{|l}
$\mathrm{M}$ \\
$\mathrm{RM}$ \\
$\mathrm{M}$ \\
$\mathrm{R}$ \\
$\mathrm{RM}$ \\
\end{tabular} \\
\hline & & $\begin{array}{l}\text { Scopacidae } \\
(3 \text { Genera })\end{array}$ & $\begin{array}{l}\text { Actitishypoleucos } \\
\text { Limosalimosa } \\
\text { Tringaglareola } \\
\text { Tringanebularia } \\
\text { Tringaocrophus } \\
\text { Tringatotanus } \\
\end{array}$ & $\begin{array}{l}\text { Common Sandpiper } \\
\text { Black-tailed Godwit } \\
\text { Wood Sandpiper } \\
\text { Common Greenshank } \\
\text { Green Sandpiper } \\
\text { Common Redshank } \\
\end{array}$ & $\begin{array}{l}\mathrm{M} \\
\mathrm{RM} \\
\mathrm{RM} \\
\mathrm{M} \\
\mathrm{R} \\
\mathrm{M} \\
\end{array}$ \\
\hline & & Glareolidae (1 Genus) & Glareolalactea & Small Pratincole & RM \\
\hline & & Scolopacidae (1 Genus) & Gallinagogallinago & Common Snipe & $\mathrm{M}$ \\
\hline & & Recurvirostridae (1 Genus) & Himantopushimantopus & Black-winged Stilt & RM \\
\hline & & $\begin{array}{l}\text { Laridae } \\
(2 \text { Genera })\end{array}$ & $\begin{array}{l}\text { Chlidoniashybrida } \\
\text { Sterna acuticauda } \\
\text { Sterna aurantia } \\
\end{array}$ & $\begin{array}{l}\text { Whiskered Tern } \\
\text { Black-bellied Tern } \\
\text { River Tern }\end{array}$ & $\begin{array}{l}\mathrm{RM} \\
\mathrm{RM} \\
\mathrm{R} \\
\end{array}$ \\
\hline 9. & $\begin{array}{l}\text { Columbiformes } \\
\text { (1 Family) }\end{array}$ & $\begin{array}{l}\text { Columbidae } \\
\text { (3 Genera) }\end{array}$ & $\begin{array}{l}\text { Columba livia } \\
\text { Streptopeliachinensis } \\
\text { Streptopeliadecaocto } \\
\text { Streptopeliaorientalis } \\
\text { Streptopeliasenegalensis } \\
\text { Streptopeliatranquebarica } \\
\text { Treronphoenicoptera } \\
\end{array}$ & $\begin{array}{l}\text { Blue rock Pigeon } \\
\text { Spotted Dove } \\
\text { Eurasian Collared Dove } \\
\text { Oriental Turtle Dove } \\
\text { Little Brown Dove } \\
\text { Red Collared Dove } \\
\text { Yellow-footed Green Pigeon } \\
\end{array}$ & \begin{tabular}{|l}
$\mathrm{R}$ \\
$\mathrm{R}$ \\
$\mathrm{R}$ \\
$\mathrm{RM}$ \\
$\mathrm{R}$ \\
$\mathrm{RM}$ \\
$\mathrm{RM}$ \\
\end{tabular} \\
\hline 10. & $\begin{array}{l}\text { Psittaciformes } \\
\text { (1 Family) }\end{array}$ & $\begin{array}{l}\text { Psittacidae } \\
\text { (1 Genus) }\end{array}$ & $\begin{array}{l}\text { Psittaculacyanocephala } \\
\text { Psittaculaeupatria } \\
\text { Psittaculakrameri }\end{array}$ & $\begin{array}{l}\text { Plum-headed Parakeet } \\
\text { Alexandrine Parakeet } \\
\text { Rose ringed Parakeet }\end{array}$ & $\begin{array}{l}\text { RM } \\
\text { RM } \\
\text { RM }\end{array}$ \\
\hline 11. & $\begin{array}{l}\text { Cuculiformes } \\
\text { (1 Family) }\end{array}$ & $\begin{array}{l}\text { Cuculidae } \\
\text { (7 Genera) }\end{array}$ & $\begin{array}{l}\text { Cacomantispasserinus } \\
\text { Centropussinensis }\end{array}$ & $\begin{array}{l}\text { Indian palaintive Cuckoo } \\
\text { Greater Coucal }\end{array}$ & $\begin{array}{l}\mathrm{RM} \\
\mathrm{R}\end{array}$ \\
\hline
\end{tabular}




\begin{tabular}{|c|c|c|c|c|c|}
\hline S. N. & Order & Family & Scientific Name & Common Name & Status \\
\hline & & & $\begin{array}{l}\text { Clamatorjacobinus } \\
\text { Cuculuscanorus } \\
\text { Cuculusmicropterus } \\
\text { Cuculusvarius } \\
\text { Eudynamysscolopacea } \\
\text { Phaenicophaeusleschenaultii } \\
\text { Surniculuslugubris } \\
\end{array}$ & $\begin{array}{l}\text { Pied Crested Cuckoo } \\
\text { Common Cuckoo } \\
\text { Indian Cuckoo } \\
\text { Common Hawk Cuckoo } \\
\text { Asian Koel } \\
\text { SirkeerMalkoha } \\
\text { Asian Drongo Cuckoo } \\
\end{array}$ & $\begin{array}{l}\text { RM } \\
\text { R } \\
\text { RM } \\
\text { RM } \\
\text { RM } \\
\text { RM } \\
\text { RM } \\
\end{array}$ \\
\hline \multirow[t]{2}{*}{12.} & \multirow{2}{*}{$\begin{array}{l}\text { Strigiformes } \\
\text { (2 Families) }\end{array}$} & Tytonidae (1 Genus) & Tyto alba & Barn Owl & $\mathrm{R}$ \\
\hline & & Strigidae (1 Genus) & Athenebrama & Spotted Owlet & $\mathrm{R}$ \\
\hline 13. & $\begin{array}{l}\text { Caprimulgiformes } \\
\text { (1 Family) }\end{array}$ & Caprimulgidae (1 Genus) & Caprimulgusasiaticus & Indian Nightjar & $\mathrm{R}$ \\
\hline \multirow[t]{2}{*}{14.} & \multirow{2}{*}{$\begin{array}{l}\text { Apodiformes } \\
\text { ( } 2 \text { Families) }\end{array}$} & Apodidae (1 Genus) & Cypsiurusbalasiensis & Asian Palm Swift & $\mathrm{R}$ \\
\hline & & Hemiprocnidae (1 Genus) & Hemiprocnecoronata & Crested Treeswift & $\mathrm{R}$ \\
\hline \multirow[t]{5}{*}{15.} & \multirow[t]{5}{*}{$\begin{array}{l}\text { Coraciiformes } \\
\text { (5 Families) }\end{array}$} & $\begin{array}{l}\text { Alcedinidae } \\
(3 \text { Genera })\end{array}$ & $\begin{array}{l}\text { Alcedoatthis } \\
\text { Cerylerudis } \\
\text { Halcyon smyrnensis }\end{array}$ & $\begin{array}{l}\text { Small Blue Kingfisher } \\
\text { Pied Kingfisher } \\
\text { White-breasted Kingfisher } \\
\end{array}$ & $\begin{array}{l}\mathrm{R} \\
\mathrm{R} \\
\mathrm{R}\end{array}$ \\
\hline & & $\begin{array}{l}\text { Meropidae } \\
(1 \text { Genus })\end{array}$ & $\begin{array}{l}\text { Meropsorientalis } \\
\text { Meropsphilippinus } \\
\end{array}$ & $\begin{array}{l}\text { Small Bee-eater } \\
\text { Blue-tailed Bee-eater } \\
\end{array}$ & $\begin{array}{l}\mathrm{R} \\
\mathrm{R}\end{array}$ \\
\hline & & Upupidae (1 Genus) & Upupaepops & Common Hoopoe & $\mathrm{R}$ \\
\hline & & Coraciidae (1 Genus) & Coraciasbenghalensis & Indian Roller & $\mathrm{R}$ \\
\hline & & Bucerotidae (1 Genus) & Ocycerosbirostris & Grey Hornbill & $\mathrm{R}$ \\
\hline \multirow[t]{2}{*}{16.} & \multirow{2}{*}{$\begin{array}{l}\text { Piciformes } \\
\text { (2 Families) }\end{array}$} & Capitonidae (1 Genus) & Megalaimahaemacephala & Crimson-breasted Barbet & $\mathrm{R}$ \\
\hline & & Picidae (3 Genera) & $\begin{array}{l}\text { Dendrocoposmahrattensis } \\
\text { Dinopiumbenghalense } \\
\text { Jynxtorquilla }\end{array}$ & $\begin{array}{l}\text { Yellow-crowned Woodpecker } \\
\text { Black-rumpedFlameback } \\
\text { Woodpecker } \\
\text { Eurasian Wryneck } \\
\end{array}$ & $\begin{array}{l}\mathrm{R} \\
\mathrm{R} \\
\mathrm{M}\end{array}$ \\
\hline \multirow[t]{9}{*}{17.} & \multirow[t]{9}{*}{$\begin{array}{l}\text { Passeriformes } \\
\text { (20 Families) }\end{array}$} & $\begin{array}{l}\text { Alaudidae } \\
\text { (6 Genera) }\end{array}$ & $\begin{array}{l}\text { Alaudagulgula } \\
\text { Ammomanesphoenicura } \\
\text { Calandrellabrachydactyla } \\
\text { Eremopterixgriseus } \\
\text { Galerida deva } \\
\text { Mirafraassamica } \\
\text { Mirafracantillans } \\
\text { Mirafraerythroptera } \\
\end{array}$ & $\begin{array}{l}\text { Oriental Skylark } \\
\text { Rufous-tailed Finch Lark } \\
\text { Greater Short-toed Lark } \\
\text { Ashy-crowned Sparrow Lark } \\
\text { Syke's Crested Lark } \\
\text { Bengal Bushlark } \\
\text { Singing Bushlark } \\
\text { Red-winged Bushlark } \\
\end{array}$ & $\begin{array}{l}\mathrm{RM} \\
\mathrm{R} \\
\mathrm{RM} \\
\mathrm{R} \\
\mathrm{R} \\
\mathrm{R} \\
\mathrm{R} \\
\mathrm{R} \\
\end{array}$ \\
\hline & & $\begin{array}{l}\text { Hirundinidae } \\
(2 \text { Genera) }\end{array}$ & $\begin{array}{l}\text { Hirundoconcolor } \\
\text { Hirundodaurica } \\
\text { Hirundofluvicola } \\
\text { Hirundorustica } \\
\text { Hirundosmithii } \\
\text { Ripariapaludicola } \\
\end{array}$ & $\begin{array}{l}\text { Dusky Crag Martin } \\
\text { Red-rumped Swallow } \\
\text { Streak-throated Swallow } \\
\text { Common Swallow } \\
\text { Wire-tailed Swallow } \\
\text { Plain Sand Martin } \\
\end{array}$ & $\begin{array}{l}\mathrm{R} \\
\mathrm{RM} \\
\mathrm{R} \\
\mathrm{RM} \\
\mathrm{R} \\
\mathrm{RM}\end{array}$ \\
\hline & & $\begin{array}{l}\text { Dicruridae } \\
\text { (1 Genus) }\end{array}$ & $\begin{array}{l}\text { Dicrurusleucophaeus } \\
\text { Dicrurusmacrocercus } \\
\text { Dicrurusparadiseus } \\
\end{array}$ & $\begin{array}{l}\text { Ashy Drongo } \\
\text { Black Drongo } \\
\text { Greater Racket-tailed Drongo }\end{array}$ & $\begin{array}{l}\mathrm{RM} \\
\mathrm{R} \\
\mathrm{RM} \\
\end{array}$ \\
\hline & & $\begin{array}{l}\text { Laniidae } \\
\text { (1 Genus) }\end{array}$ & $\begin{array}{l}\text { Laniuscristatus } \\
\text { Laniusschach } \\
\text { Laniusvittatus } \\
\end{array}$ & $\begin{array}{l}\text { Brown Shrike } \\
\text { Rufous Backed Shrike } \\
\text { Bay-backed Shrike }\end{array}$ & $\begin{array}{l}\mathrm{RM} \\
\mathrm{R} \\
\mathrm{RM} \\
\end{array}$ \\
\hline & & Oriolidae (1 Genus) & Oriolusoriolus & Eurasian Golden Oriole & $\mathrm{R}$ \\
\hline & & $\begin{array}{l}\text { Sturnidae } \\
(2 \text { Genera })\end{array}$ & \begin{tabular}{|l|} 
Acridotherestristis \\
Sturnus contra \\
Sturnuspagodarum \\
Sturnusroseus
\end{tabular} & $\begin{array}{l}\text { Common Myna } \\
\text { Asian Pied Starling } \\
\text { Brahminy Starling } \\
\text { Rosy Starling } \\
\end{array}$ & $\begin{array}{l}\mathrm{R} \\
\mathrm{R} \\
\mathrm{R} \\
\mathrm{RM} \\
\end{array}$ \\
\hline & & $\begin{array}{l}\text { Corvidae } \\
(2 \text { Genera) }\end{array}$ & $\begin{array}{l}\text { Corvusmacrorhynchos } \\
\text { Corvussplendens } \\
\text { Dendrocittavagabunda } \\
\end{array}$ & $\begin{array}{l}\text { Jungle Crow } \\
\text { House Crow } \\
\text { RufousTreepie } \\
\end{array}$ & $\begin{array}{l}\mathrm{R} \\
\mathrm{R} \\
\mathrm{R}\end{array}$ \\
\hline & & $\begin{array}{l}\text { Campephagidae } \\
(2 \text { Genera) }\end{array}$ & $\begin{array}{l}\text { Coracinamelanoptera } \\
\text { Pericrocotuscinnamomeus }\end{array}$ & $\begin{array}{l}\text { Black-headed Cuckooshrik } \\
\text { Small Minivet }\end{array}$ & $\begin{array}{l}\mathrm{R} \\
\mathrm{R} \\
\end{array}$ \\
\hline & & $\begin{array}{l}\text { Irenidae } \\
(2 \text { Genera })\end{array}$ & $\begin{array}{l}\text { Aegithinatiphia } \\
\text { Pycnonotuscafer } \\
\text { Pycnonotusluteolus }\end{array}$ & $\begin{array}{l}\text { Common Iora } \\
\text { Red-vented Bulbul } \\
\text { White-browed Bulbul } \\
\end{array}$ & \begin{tabular}{|l|}
$\mathrm{R}$ \\
$\mathrm{R}$ \\
$\mathrm{RM}$ \\
\end{tabular} \\
\hline
\end{tabular}




\begin{tabular}{|c|c|c|c|c|c|}
\hline \multirow[t]{13}{*}{ S. N. } & Order & Family & Scientific Name & Common Name & Status \\
\hline & & $\begin{array}{l}\text { Timaliidae } \\
\text { (3 Genera) }\end{array}$ & $\begin{array}{l}\text { Chrysommasinense } \\
\text { Dumetiahyperythra } \\
\text { Turdoidescaudata } \\
\text { Turdoidesmalcolmi } \\
\text { Turdoidesstriata }\end{array}$ & $\begin{array}{l}\text { Yellow-eyed Babbler } \\
\text { Tawny-bellied Babbler } \\
\text { Common Babbler } \\
\text { Large Grey Babbler } \\
\text { Jungle Babbler }\end{array}$ & $\begin{array}{l}\mathrm{R} \\
\mathrm{RM} \\
\mathrm{R} \\
\mathrm{R} \\
\mathrm{R}\end{array}$ \\
\hline & & $\begin{array}{l}\text { Monarchidae } \\
\text { (3 Genera) }\end{array}$ & $\begin{array}{l}\text { Hypothymisazurea } \\
\text { Rhipiduraalbicollis } \\
\text { Rhipiduraaureola } \\
\text { Terpsiphoneparadisi }\end{array}$ & $\begin{array}{l}\text { Black-naped Monarch } \\
\text { White-throated Fantail } \\
\text { White-browed Fantail } \\
\text { Asian Paradise-flycatcher }\end{array}$ & $\begin{array}{l}\mathrm{RM} \\
\mathrm{R} \\
\mathrm{R} \\
\mathrm{RM} \\
\end{array}$ \\
\hline & & $\begin{array}{l}\text { Muscicapidae } \\
\text { (2 Genera) }\end{array}$ & $\begin{array}{l}\text { Cyornistickelliae } \\
\text { Muscicapadauurica }\end{array}$ & $\begin{array}{l}\text { Tickell's Blue Flycatcher } \\
\text { Asian Brown Flycatcher }\end{array}$ & $\begin{array}{l}\mathrm{R} \\
\mathrm{RM}\end{array}$ \\
\hline & & $\begin{array}{l}\text { Sylviidae } \\
\text { (5 Genera) }\end{array}$ & $\begin{array}{l}\text { Acrocephalusdumetorum } \\
\text { Hippolaiscaligata } \\
\text { Orthotomussutorius } \\
\text { Phylloscopustrochiloides } \\
\text { Priniainornata } \\
\text { Priniasocialis } \\
\text { Priniasylvatica } \\
\end{array}$ & $\begin{array}{l}\text { Blyth's Reed Warbler } \\
\text { Booted Warbler } \\
\text { Common Tailorbird } \\
\text { Greenish Warbler } \\
\text { Plain Prinia } \\
\text { Ashy Prinia } \\
\text { Jungle Prinia } \\
\end{array}$ & $\begin{array}{l}\mathrm{RM} \\
\mathrm{RM} \\
\mathrm{R} \\
\mathrm{RM} \\
\mathrm{R} \\
\mathrm{R} \\
\mathrm{RM} \\
\end{array}$ \\
\hline & & $\begin{array}{l}\text { Turdidae } \\
\text { (7 Genera) }\end{array}$ & $\begin{array}{l}\text { Cercomelafusca } \\
\text { Copsychussaularis } \\
\text { Lusciniasvecica } \\
\text { Phoenicurusochruros } \\
\text { Saxicolacaprata } \\
\text { Saxicoloidesfulicata } \\
\text { Zootheracitrina } \\
\end{array}$ & $\begin{array}{l}\text { Brown Rock-Chat } \\
\text { Oriental Magpie Robin } \\
\text { Bluethroat } \\
\text { Black Redstart } \\
\text { Pied Bushchat } \\
\text { Indian Robin } \\
\text { Orange-headed Thrush }\end{array}$ & $\begin{array}{l}\mathrm{R} \\
\mathrm{R} \\
\mathrm{RM} \\
\mathrm{RM} \\
\mathrm{R} \\
\mathrm{R} \\
\mathrm{R} \\
\end{array}$ \\
\hline & & $\begin{array}{l}\text { Motacillidae } \\
(2 \text { Genera })\end{array}$ & \begin{tabular}{|l|} 
Anthusrufulus \\
Anthussimilis \\
Motacilla alba \\
Motacillacineara \\
Motacillacitreola \\
Motacillaflava \\
Motacillamaderaspatensis
\end{tabular} & $\begin{array}{l}\text { Paddy field Pipit } \\
\text { Long-billed Pipit } \\
\text { White Wagtail } \\
\text { Grey Wagtail } \\
\text { Citrine Wagtail } \\
\text { Yellow Wagtail } \\
\text { Large Pied Wagtail } \\
\end{array}$ & $\begin{array}{l}\mathrm{R} \\
\mathrm{RM} \\
\mathrm{RM} \\
\mathrm{RM} \\
\mathrm{RM} \\
\mathrm{RM} \\
\mathrm{R} \\
\end{array}$ \\
\hline & & Zosteropidae (1 Genus) & Zosteropspalpebrosus & Oriental White-Eye & $\mathrm{R}$ \\
\hline & & \begin{tabular}{|l|} 
Dicaeidae (1 Genus) \\
\end{tabular} & Dicaeumerythrorynchos & Pale-billed Flowerpecker & $\mathrm{R}$ \\
\hline & & $\begin{array}{l}\text { Nectarinidae } \\
(1 \text { Genus })\end{array}$ & $\begin{array}{l}\text { Nectariniaasiatica } \\
\text { Nectariniazeylonica }\end{array}$ & $\begin{array}{l}\text { Purple Sunbird } \\
\text { Purple-rumped Sunbird }\end{array}$ & $\begin{array}{l}\mathrm{R} \\
\mathrm{R}\end{array}$ \\
\hline & & $\begin{array}{l}\text { Ploceidae } \\
\text { (2 Genera) }\end{array}$ & $\begin{array}{l}\text { Passer domesticus } \\
\text { Ploceusphilippinus }\end{array}$ & $\begin{array}{l}\text { House Sparrow } \\
\text { Baya Weaver }\end{array}$ & $\begin{array}{l}\mathrm{R} \\
\mathrm{RM}\end{array}$ \\
\hline & & $\begin{array}{l}\text { Estrildidae } \\
(2 \text { Genera })\end{array}$ & $\begin{array}{l}\text { Amandavaamandava } \\
\text { Lonchuramalabarica } \\
\text { Lonchuramalacca } \\
\text { Lonchurrapunctulata }\end{array}$ & $\begin{array}{l}\text { Red Munia } \\
\text { Indian Silverbill } \\
\text { Black-headed Munia } \\
\text { Scaly-breasted Munia } \\
\end{array}$ & $\begin{array}{l}\mathrm{RM} \\
\mathrm{R} \\
\mathrm{R} \\
\mathrm{R} \\
\end{array}$ \\
\hline & Orders- 17 & Families- 51 & No. of Bird Genera- 128 & No. of Bird species- 190 & \\
\hline
\end{tabular}

R- Resident; RM- Resident Migrant; M- Migrant. 
Table 3: Distribution of avifauna in respective orders and families

\begin{tabular}{|l|l|c|c|c|}
\hline S.N. & Order & No. of Families & No. of Genera & No. of Species \\
\hline 1. & Podicipediformes & 01 & 02 & 02 \\
\hline 2. & Pelecaniformes & 01 & 02 & 04 \\
\hline 3. & Ciconiiformes & 03 & 15 & 19 \\
\hline 4. & Anseriformes & 01 & 07 & 16 \\
\hline 5. & Falconiformes & 01 & 08 & 10 \\
\hline 6. & Galliformes & 01 & 03 & 04 \\
\hline 7. & Gruiformes & 01 & 04 & 05 \\
\hline 8. & Charadriiformes & 07 & 12 & 19 \\
\hline 9. & Columbiformes & 01 & 03 & 07 \\
\hline 10. & Psittaciformes & 01 & 01 & 03 \\
\hline 11. & Cuculiformes & 01 & 07 & 09 \\
\hline 12. & Strigiformes & 02 & 02 & 02 \\
\hline 13. & Caprimulgiformes & 01 & 01 & 01 \\
\hline 14. & Apodiformes & 02 & 02 & 02 \\
\hline 15. & Coraciiformes & 05 & 07 & 08 \\
\hline 16. & Piciformes & 02 & 04 & 04 \\
\hline 17. & Passeriformes & 20 & 48 & 75 \\
\hline & $\mathbf{1 7}$ & $\mathbf{5 1}$ & $\mathbf{1 2 8}$ & $\mathbf{1 9 0}$ \\
\hline
\end{tabular}

The maximum bird species are belonging to the order Passeriformes $(39.47 \%)$ followed by Ciconiiformes and Charadriiformes (10.00\%); Anseriformes (8.42\%); Falconiformes (5.26\%); Coraciiformes (4.21\%); Cuculiformes (4.74\%); Columbiformes (3.68\%); Gruiformes (2.63\%); Pelecaniformes, Galliformes and Piciformes (2.11\%), Psittaciformes (1.58\%); Podicipediformes, Strigiformes and Apodiformes $(1.05 \%)$ and minimum species reported in order Caprimulgiformes $(0.53 \%)$ (Figure 1$)$.

Figure 1: To show avian species percentage in respective orders

\section{Avian species \% in respective orders}

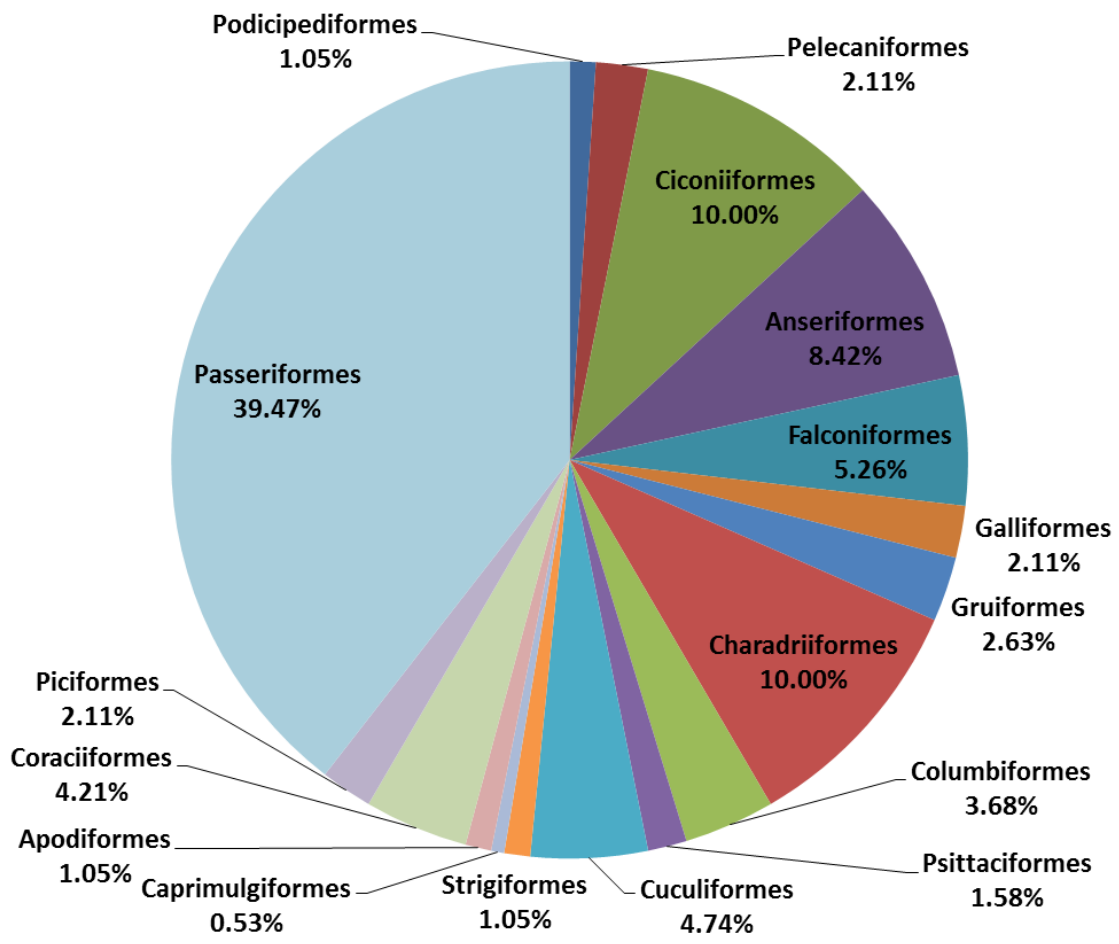

These 17 orders are divided in 51 families. The largest number of families were reported in order Passeriformes (20) followed by Charadriiformes (07); Coraciiformes (05); Ciconiiformes (03); Strigiformes, Apodiformes and Piciformes (02); and only single family reported in order Podicipediformes, Pelecaniformes, Anseriformes, Falconiformes, Galliformes, Gruiformes,Columbiformes, Psittaciformes, Cuculiformes and Caprimulgiformes (Figure 2, Table 3). 
Figure2: To show avian species and family diversity in respective orders

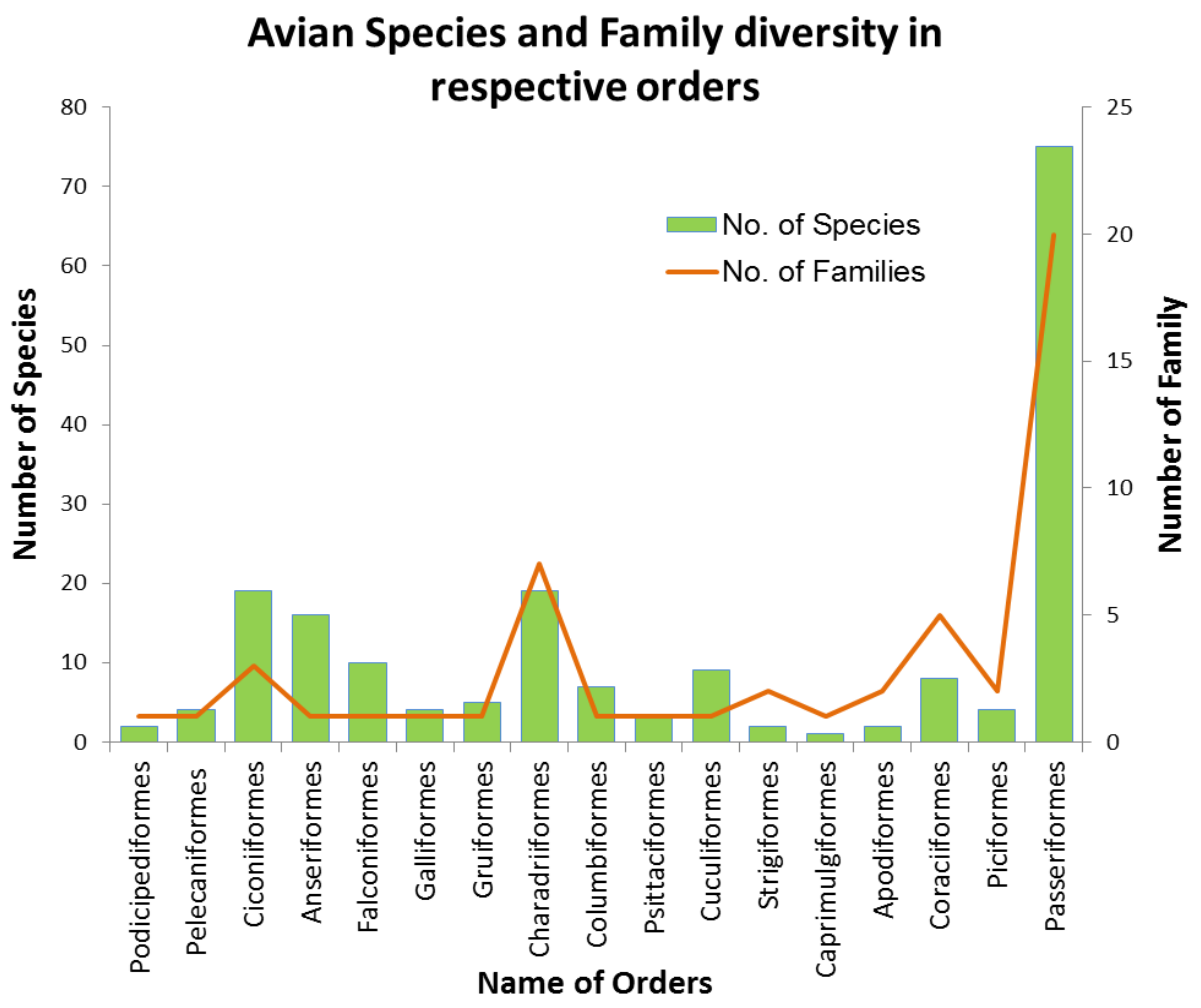

In the present study out of total 190 bird species, $89(46.84 \%)$ are resident, $77(40.53 \%)$ are resident migrant and 24 $(12.63 \%)$ are migratory species (Table 2 ).

Table 2: Status of Avifauna of Gorewada International Bio-Park.

\begin{tabular}{|l|l|c|c|}
\hline S.N. & Avian species & No. of Avian species & \% of Avian species \\
\hline 1. & Resident & 89 & 46.84 \\
\hline 2. & Resident Migrant & 77 & 40.53 \\
\hline 3. & Migrant & 24 & 12.63 \\
\hline & & $\mathbf{1 9 0}$ & 100.00 \\
\hline
\end{tabular}

\section{Discussion}

In Gorewada International Park, Nagpur we have recorded 190 species belonging to 128 genera, 51 families and 17 orders. Out of 190bird species, 89(46.84\%) species are resident, $77(40.53 \%)$ are resident migrant and $24(12.63 \%)$ were migratory species. The order Passeriformes is the largest order which covered 20 families out of 51. Patil and Tijare (2012) were recorded 82 bird species from 11 orders and 36 families in the vicinity of Gorewadalake, Nagpur (Maharashtra). Maximum species were observed during monsoon and winter months in compare to summer months probably due to scarcity of food. They also reported 72 species belonging to 12 orders and 35 families from Borgaon near Gorewada (Patil and Tijare 2013).

Chinchkhede and Kedar (2012b) were counted 59 species of birds of which 45 were resident, 08 were winter migrants, 04 were local migrants and 02 were found to be summer migrants in Sringarlake, near Navegaon national park. In and around Koradilake of Nagpur, they were reported 76 species belonging to 15 orders among which 54 species were resident; 09 species were seasonal local migrant and 13 species were winter migrant. There was a gradual decline in species richness as the weather condition changes from colder to warmer. Order Passeriformes (26 species) dominated the avifauna of the Koradilake which was followed by order Ciconiiformes (10species). The minimum diversity was recorded in the months of monsoon due to heavy rain, increased flow of water, nonavailability of food and return of migratory birds (Chinchkhede and Kedar, 2012a).

While, in and around Ambazarilake, Kedar (2012) was observed 135 species of birds out of which 105 species were recorded as resident, 17 species were resident migrant and 13 were winter migrant. In Bamanwada Lake of Rajura, Chilke (2012) was recorded 58 species of birds belonging to 9 orders and 29 families. Among 9 orders Passeriformes consists of $34.48 \%$ of total families out of 29 . 
The above observations are similar to the present study where, residents birds are predominant than that of resident migrant and migratory birds; the number of birds are more during winter and Least number of birds are reported during summer and Passeriformes is the dominating order of birds.

\section{CONCLUSION}

Gorewada International bio-park is covered by dense forest on three sides of the lake and provides heterogeneity in vegetation for avian species. The importance of the lake is as a major water source and the forest around it have prevented the lake's decline. This lake is rich in aquatic fauna which includes micro and macro-organisms, shrimps, fishes and protein-rich invertebrates and other food. This region is suitable for feeding, resting and roosting due to the abundance of food, accessibility to food resources, availability of exposed mudflats and shorelines. Gorewada Bio-park is an important site for many resident and migratory species of birds which are excellent indicators of ecological health. Therefore, the conservation of this bio-park is very essential to sustain resident and migratory bird populations along with the threatened and vulnerable birds of this area.

This study will be helpful to obtain comprehensive information regarding staging areas of migratory birds, feeding areas of resident migratory birds and breeding areas of resident birds. This study will also be helpful for protection and conservation of birds.

\section{REFERENCES}

Ali Salim (2001) The book of Indian birds, Thirteenth Revised Edition, BNHS, Oxford University Press, Mumbai.

Chilke A. M. (2012) Avian Diversity in and around Bamanwada Lake of Rajura, District- Chandrapur (Maharashtra) Annals of Biological Research, 3 (4):2014-2018.

Chinchkhede K. H. and Kedar G. T. (2012a) Avifaunal diversity of Koradi Lake in Nagpur District of central India. Journal of Research in Biology, 2:070-076.

Chinchkhede K. H. and Kedar G. T. (2012b) Quantitative analysis of avifauna of Sringarlake, near Navegaon national park, Maharashtra. IOSR Journal of Pharmacy, 2(6):09-16.

Gregory R. D., Gibbons D. W. and DonaldP. F. (2004) Bird census and survey techniques.

Grimmett R., Inskipp C. and Inskipp T. (1998) Birds of the Indian Subcontinent. London: Christopher Helm.

Kedar G.T. (2012) Ambazarilake - A potential stopover point of winter migrants in Central India. Indian Streams Research Journal, 2(6):1-8.

Padmavati A, Alexandar R, Anbarashan M. (2010) Our Nature 8:247-253.

Patil, K. G. and Tijare R. V. (2012).Study of Feeding Habits of Avifauna in Vicinity of Gorewada Lake, Nagpur, Maharashtra (India). Bionano Frontier Special issue 9:112-114.

Patil, K. G. and Tijare R. V. (2013). Chapter-28. "Study of Avifauna in Visited to Borgaon Region of Nagpur, Maharashtra, India." In Impact of Global Climate Change on Earth Ecosystems. (Khanna D. R. et al. Edis.) Biotech Books New Delhi. Pp 223-240.

Sanjay G. S. (1993) An ecological study of birds at Kokkare Bellur. WWF-India, New Delhi. Final Report. 